Article

\title{
Sharpe's Ratio for Oriented Fuzzy Discount Factor
}

\author{
Anna Łyczkowska-Hanćkowiak \\ Department of Finance, WSB University in Poznań, 61-895 Poznań, Poland; \\ anna.lyczkowska-hanckowiak@wsb.poznan.pl
}

Received: 10 January 2019; Accepted: 12 March 2019; Published: 16 March 2019

\begin{abstract}
The analysis presented in this paper regards the security of a present value given as an ordered fuzzy number. The present value was estimated in an imprecise manner and supplemented by the forecast of its coming changes. A discount factor of such security is an ordered fuzzy number of the orientation identical to the oriented present value that determines it. All classical methods of portfolio analysis are based on the definition of the return rate. In the case of securities with a fuzzy present value, a discount factor is a better tool for portfolio analysis than the return rate, which implies the chosen methods of management of securities should be revised and transformed to equivalent methods based on a discount factor. This would enable the use of those methods in the case of a financial instrument of the oriented fuzzy present value. This paper presents example results of the realization of such a postulate. The main aim of the paper is to generalize Sharpe's ratio to a case of investment recommendations management formulated for a security characterized by an oriented discount factor. A five-degree rating scale was used. The whole deliberation is illustrated by broad numerical examples.
\end{abstract}

Keywords: Sharpe's ratio; ordered fuzzy number; fuzzy oriented discount factor

\section{Introduction}

An imprecise number is a family of values in which each considered value belongs to it to a varying degree. A commonly accepted model of imprecise numbers is the fuzzy number (FN), defined as a fuzzy subset of the real line $\mathbb{R}$. The most general definition of FN was given by Dubois and Prade [1].

Ordered fuzzy numbers (OFN) were defined in an intuitive way by Kosiński and his collaborators who, in this way, introduced a fuzzy number supplemented by the orientation [2-5]. A positive orientation means the expectation of value growth, while a negative one forecasts a decrease in the value. The competent elaboration on the current state-of-the-art on OFNs is the monograph [6]. Kosiński showed that there exist OFNs that are not fuzzy numbers [5]. Thus, Kosiński's original theory is revised in [7].

By a security, we understand an authorization to receive a future financial revenue, payable at a certain maturity. The value of this revenue is interpreted as an anticipated future value (FV) of the asset. We can point out a time in the future when the considered income value will already be known to the observer. This, together with the Kolmogorov's postulate $[8,9]$, leads to the conclusion that FV is a random variable.

The reference point for appraising the financial asset is its present value (PV), defined as a present equivalent of a payment available at a given time in the future [10]. It is commonly accepted that the PV of future cash flows is an approximate value. The natural consequence of this approach is estimating PV with fuzzy numbers. This is reflected in defining a fuzzy PV as a discounted fuzzy forecast of a future cash flow's value [11]. The concept of using fuzzy numbers in financial arithmetic was reported by Buckley [12]. Ward's definition is generalized in [13] to the case of imprecisely assessed postponement. Sheen [14] expanded Ward's definition to the case of fuzzy nominal interest rate. 
Buckley [12], Gutierez [15], Kuchta [16] and Lesage [17] discussed the problems connected to applying fuzzy arithmetic and calculating fuzzy PV. They previously proved the sensibility of using trapezoidal fuzzy numbers as a fuzzy financial arithmetic tool. Huang [18] expanded Ward's definition even further, to the case of future cash flow given as a fuzzy variable. A more general definition of fuzzy PV was proposed by Tsao [19], who assumed that future cash flow can be treated as a fuzzy probabilistic set. Calzi [20] formulated an axiomatic definition of fuzzy PV. All these authors depicted PV as a discount of an imprecisely estimated future cash flow value. A different approach is introduced in [21], where the fuzzy PV is estimated based on the current market price of a financial asset. Additionally, some applications of fuzzy PV are discussed in [22-25].

The basis of any security assessment is the return rate, generally defined as any nonincreasing function of PV and nondecreasing function of FV. Both financial theorists and practitioners notice the problem of imprecise return rates assessment and the problem of imposed imprecise limitations. Meanwhile, rapid development of fuzzy system mathematics leads to the creation of fuzzy portfolio analysis as well as many fuzzy portfolio models. The main idea of this approach is to apply existing portfolio theory and then fuzzify some of the considered parameters, such as return rate, present value [26-28] or probability distribution parameters [29].

Fuzzy systems mathematics are also used in quantitative finance when uncertainty is modeled with a fuzzy number. In most papers regarding this notion, it is assumed in advance that the return rate from a financial instrument is a fuzzy set [30-35].

However, this assumption is generally connected to uncertainty of the return rate and unclear or incomplete information used by the investor. To model a financial asset understood in this way, researchers mostly applied possibility theory [36-38] and credibility theory [39]. Kahraman et al. [40] introduced a study, in which both cash flows and their return rates are given as trapezoidal fuzzy numbers. This kind of approach is aimed at showing that market experts, while appointing a net present value, operate only on some imprecise values. Competent sources of information about this topic are monographies [41,42]. A common feature connecting the abovementioned models is the usage of fuzzy set membership function as a substitute for probability distribution. This means that the uncertainty researched in these models is, in fact, replaced by imprecision. This kind of research postulate was first formulated by Kosko [43].

In most of the proposed models, inclusion of imprecision is an a priori assumption given fuzzy return rates from financial instruments. In this article, the source of the fuzziness is an imprecisely given present value. Piasecki $[21,25]$ noticed that, due to imprecise appointment of a present value and considering the future value as a variable, it is possible to describe the return rate of an instrument as a fuzzy probabilistic set [44]. The proposed model not only includes the imprecision problem but also indicates the existence of uncertainty burdening a financial asset.

The works in $[18,25,45-48]$ do not use the research postulate formulated by Kosko, because the membership function in these models does not replace the probability distribution, but only interacts with it as a distinct entity. This kind of model extension significantly enhances the possibilities of a reliable return rate description. Despite encompassing the imprecise information in the return rate assessment, in the proposed fuzzy model, the whole existing empirical knowledge about return rate probability distribution can be used without any further amendments. This feature is highly beneficial, especially by allowing realistic applications of the model. Moreover, in these models, the uncertainty interacts with imprecision, which agrees with the research paradigm formulated by Hiroto [44].

If PV is evaluated by a fuzzy number, then the expected return rate is obtained as a fuzzy subset in the real line. This result is the theoretical foundation for investment strategies presented in [49].

The main aim of the paper is the analysis of the possibility to expand the above-mentioned investment strategies to a case when the PV is examined via ordered fuzzy numbers. To fulfill that task, Sharpe's ratio [50] is extended for that case. In the original Sharpe's ratio, the main premise for formulating investment recommendation is the expected return rate of the analyzed security. On the other hand, in [47], it is shown that the expected fuzzy discount factor is a better tool for appraising 
considered securities than the fuzzy expected return rate. It is shown that the use of the expected fuzzy discount factor significantly facilitates the portfolio analysis. For this reason, the original Sharpe's ratio is transformed to an equivalent form, in which the basic premise to form investment recommendation is the expected fuzzy discount factor for the analyzed securities.

This paper is organized as follows. Section 2 presents the basic definitions and characteristics of OFNs. Section 3 briefly describes imprecision and its assessment. Sections 4 and 5 briefly discuss oriented fuzzy present value and oriented fuzzy discount factor, respectively. In Section 6, a five-degree scale of investment recommendation is presented. Section 6 describes Sharpe's ratio for the oriented discount factor. This enables using a modified criterion for investment recommendations management. Section 7 includes a case study that illustrates the use of predefined Sharpe's ratio for management of investment recommendations with the present value given as trapezoidal oriented fuzzy number. Section 8 is a brief summary.

\section{Ordered Fuzzy Numbers-Basic Facts}

By $\mathcal{F}(\mathbb{X})$, we denote the family of all fuzzy subsets of a space $\mathbb{X}$. An imprecise number is a family of values in which each considered value belongs to it to a varying degree. A commonly accepted model of the imprecise number is the fuzzy number (FN), defined as a fuzzy subset of the real line $\mathbb{R}$. The most general definition of FN was given by Dubois and Prade [1]. A fuzzy number is defined in the following manner:

Definition 1. For any $F N \mathcal{L}$, there exists a nondecreasing sequence $(a, b, c, d) \subset \mathbb{R}$ such that $\mathcal{L} \in \mathcal{F}(\mathbb{R})$ is represented by its membership function $\mu_{\mathcal{L}}\left(\cdot \mid a, b, c, d, L_{L}, R_{L}\right) \in[0 ; 1]^{\mathbb{R}}$ given by the identity:

$$
\mu_{\mathcal{L}}\left(x \mid a, b, c, d, L_{L}, R_{L}\right)= \begin{cases}0, & x \notin[a, d], \\ L_{L}(x), & x \in[a, b], \\ 1, & x \in[b, c], \\ R_{L}(x), & x \in[c, d],\end{cases}
$$

where the left reference function $L_{L} \in[0 ; 1]^{[a, b]}$ and the right reference function $R_{L} \in[0 ; 1]^{[c, d]}$ are the upper semi-continuous monotonic functions satisfying the conditions:

$$
\begin{gathered}
L_{L}(b)=R_{L}(c)=1 \\
\forall_{x \in] a, d[}: \mu_{\mathcal{L}}\left(x \mid a, b, c, d, L_{L}, R_{L}\right)>0 .
\end{gathered}
$$

Moreover, Dubois and Prade [51] introduced such arithmetic operations on FN that are coherent with the Zadeh extension principle.

\subsection{Definition of Ordered Fuzzy Numbers}

Ordered fuzzy numbers (OFN) were intuitively introduced by Kosiński and his colleagues in a series of papers [2-5] as an extension of the concept of FN. A significant drawback of Kosiński's theory is that there exist OFNs that are not FN [5]. Kosiński's intuitive approach to the notion of OFN is very useful. The OFNs' usefulness results from the fact that an OFN is defined as FN supplemented by a negative orientation or a positive one. The negative orientation means the order is from bigger to smaller numbers. The positive orientation means the order is from smaller to bigger numbers. The FN orientation is interpreted as prediction of future FN changes. Kosiński's theory was revised by Piasecki [7]. OFNs are generally defined in the following way: 
Definition 2. Let for any monotonic sequence $(a, b, c, d)$ $\subset \mathbb{R}$ an ordered fuzzy number (OFN) $\overleftrightarrow{\mathcal{L}}\left(a, b, c, d, S_{L}, E_{L}\right)$ be defined as a pair of fuzzy numbers determined by their membership function $\mu_{\mathcal{L}}\left(\cdot \mid a, b, c, d, S_{L}, E_{L}\right) \in[0 ; 1]^{\mathbb{R}}$ given by the identity:

$$
\mu_{\mathcal{L}}\left(x \mid a, b, c, d, S_{L}, E_{L}\right)= \begin{cases}0, & x \notin[a, d]=[d, a] \\ S_{L}(x), & x \in[a, b]=[b, a] \\ 1, & x \in[b, c]=[c, b] \\ E_{L}(x), & x \in[c, d]=[d, c]\end{cases}
$$

and orientation $a \longmapsto d=(a, d)$, where the starting function $S_{L} \in[0 ; 1]^{[a, b]}$ and the ending function $E_{L} \in$ $[0 ; 1]^{[c, d]}$ are upper semi-continuous monotonic functions satisfying the conditions:

$$
\begin{gathered}
S_{L}(b)=E_{L}(c)=1 \\
\forall_{x \in] a, d[} \mu_{\mathcal{L}}\left(x \mid a, b, c, d, S_{L}, E_{L}\right)>0 .
\end{gathered}
$$

Let us note that the identity in Equation (4) describes the additionally extended notation of numerical intervals, which is used in this work.

The space of all OFN is denoted by the symbol $\mathbb{K}$. The condition $a<d$ fulfilment determines the positive orientation $a \longmapsto d$ of $\mathrm{OFN} \stackrel{\leftrightarrow}{\mathcal{L}}\left(a, b, c, d, S_{L}, E_{L}\right)$. In this case, the starting function $S_{L}$ is nondecreasing and the ending function $E_{L}$ is nonincreasing. Any positively oriented OFN is interpreted as an imprecise number that might increase. The condition $a>d$ fulfilment determines the negative orientation of OFN $\overleftrightarrow{\mathcal{L}}\left(a, b, c, d, S_{L}, E_{L}\right)$. In this case, the starting function $S_{L}$ is nonincreasing and the ending function $E_{L}$ is nondecreasing. Negatively oriented OFN is interpreted as an imprecise number that might decrease. For the case $a=d, \operatorname{OFN} \stackrel{\leftrightarrow}{\mathcal{L}}\left(a, a, a, a, S_{L}, E_{L}\right)$ represents a crisp number $a \in \mathbb{R}$, which is not oriented.

Herein, we limit our deliberations to a special case of ordered fuzzy numbers—trapezoidal ordered fuzzy numbers defined in [7] in the following manner.

Definition 3. For any monotonic sequence $(a, b, c, d) \subset \mathbb{R}$, the trapezoidal ordered fuzzy number (TrOFN) $\overleftrightarrow{\operatorname{Tr}}(a, b, c, d)$ is defined as the pair of FNs determined by their membership function $\mu \overleftrightarrow{\operatorname{Tr}}(\cdot \mid a, b, c, d) \in[0 ; 1]^{\mathbb{R}}$ given by the identity:

$$
\mu_{\overleftrightarrow{T r}}(x \mid a, b, c, d)= \begin{cases}0, & x \notin[a, d]=[d, a] \\ \frac{x-a}{b-a}, & x \in[a, b[=] b, a] \\ 1, & x \in[b, c]=[c, b] \\ \frac{x-d}{c-d}, & x \in] c, d]=[d, c[\end{cases}
$$

and orientation $a \longmapsto d$.

The space of all TrOFNs is denotes by the symbol $\mathbb{K}_{T r}$.

Kosiński has introduced the arithmetic operators of dot product $\odot$ for TrOFNs in the following way. For any real number $\beta \in \mathbb{R}$ and any $\operatorname{TrOFN} \overleftrightarrow{\operatorname{Tr}}(a, b, c, d)$, their dot product can be calculated as follows:

$$
\beta \odot \overleftrightarrow{\operatorname{Tr}}(a, b, c, d)=\overleftrightarrow{\operatorname{Tr}}(\beta \cdot a, \beta \cdot b, \beta \cdot c, \beta \cdot d)
$$

The arithmetic proposed by Kosiński has a significant disadvantage. The space of ordered fuzzy numbers is not closed under Kosiński's addition. Therefore, Kosinski's theory is modified so that the space of ordered fuzzy numbers is closed under revised arithmetic operations. The sum $\boxplus$ for TrOFNs is determined as follows [7]. In the case of any $\operatorname{TrOFNs} \overleftrightarrow{\operatorname{Tr}}(a, b, c, d)$ and $\overleftrightarrow{\operatorname{Tr}}(p-a, q-b, r-c, s-d)$, their sum is determined as follows: 


$$
\begin{gathered}
\overleftrightarrow{\operatorname{Tr}}(a, b, c, d) \boxplus \overleftrightarrow{\operatorname{Tr}}(p-a, q-b, r-c, s-d) \\
=\left\{\begin{array}{l}
\overleftrightarrow{\operatorname{Tr}}(\min \{p, q\}, q, r, \max \{r, s\}),(q<r) \vee(q=r \wedge p \leq s), \\
\overleftrightarrow{\operatorname{Tr}}(\max \{p, q\}, q, r, \min \{r, s\}),(q>r) \vee(q=r \wedge p>s) .
\end{array}\right.
\end{gathered}
$$

\subsection{Relation "Greater Than or Equal to" for Ordered Fuzzy Numbers}

In the set of trapezoidal ordered fuzzy numbers, the relation of a fuzzy preorder is determined. Let us consider the pair $(\overleftrightarrow{\mathcal{K}}, \overleftrightarrow{\mathcal{L}}) \in \mathbb{K}_{T r} \times \mathbb{K}_{T r}$. On the set $\mathbb{K}_{t r}$ of all TrOFNs, we define the relation $\overleftrightarrow{\mathcal{K}} \succcurlyeq \overleftrightarrow{\mathcal{L}}$ as follows:

$$
\overleftrightarrow{\mathcal{K}} \succcurlyeq \overleftrightarrow{\mathcal{L}} \Leftrightarrow \text { “TrOFN } \overleftrightarrow{\mathcal{K}} \text { is greater than or equal to } \operatorname{TrOFN} \stackrel{\leftrightarrow}{\mathcal{L}} . ”
$$

This relation is a fuzzy preorder $Q \in \mathcal{F}\left(\mathbb{K}_{T r} \times \mathbb{K}_{T r}\right)$ determined by means of such membership function $v_{Q} \in[0,1]^{\mathbb{K}_{T r} \times \mathbb{K}_{T r}}$ that, from the point of view of multivalued logic, the value $v_{Q}(\overleftrightarrow{\mathcal{K}}, \stackrel{\leftrightarrow}{\mathcal{L}})$ may be interpreted as true value of the sentence in Equation (10). The variability of membership function $v_{Q}$ is described in detail as follows:

Theorem 1. For any pair $(\overleftrightarrow{\mathcal{K}}, \overleftrightarrow{\mathcal{L}}) \in \mathbb{K} \times \mathbb{K}$ satisfying the condition:

$$
\overleftrightarrow{\mathcal{K}} \boxplus((-1) \odot \overleftrightarrow{\mathcal{L}})=\overleftrightarrow{\mathcal{M}}=\overleftrightarrow{\operatorname{Tr}}\left(a_{\mathcal{M}}, b_{\mathcal{M}}, c_{\mathcal{M}}, d_{\mathcal{M}}\right)
$$

we have:

- If $a_{\mathcal{M}} \leq d_{\mathcal{M}}$, then

$$
v_{Q}(\overleftrightarrow{\mathcal{K}}, \overleftrightarrow{\mathcal{L}})= \begin{cases}0, & 0>a_{\mathcal{M}} \\ \frac{-a_{\mathcal{M}}}{b_{\mathcal{M}}-a_{\mathcal{M}}}, & a_{\mathcal{M}} \leq 0<b_{\mathcal{M}} \\ 1, & b_{\mathcal{M}} \geq 0\end{cases}
$$

- If $a_{\mathcal{M}}>d_{\mathcal{M}}$, then

$$
v_{Q}(\overleftrightarrow{\mathcal{K}}, \overleftrightarrow{\mathcal{L}})= \begin{cases}0, & 0>d_{\mathcal{M}} \\ \frac{-d_{\mathcal{M}}}{c_{\mathcal{M}}-d_{\mathcal{M}}}, & d_{\mathcal{M}} \leq 0<c_{\mathcal{M}} \\ 1, & c_{\mathcal{M}} \geq 0\end{cases}
$$

\section{Imprecision Assessment}

FNs are widely used for modeling assessments or estimations of imprecisely given parameters. Following the work in [52], we understand imprecision as a superposition of ambiguity and indistinctness of information. Ambiguity can be interpreted as a lack of clear recommendation between one alternative among various others. Indistinctness is understood as a lack of explicit distinction between recommended and not recommended alternatives. An increase in information imprecision makes it less useful and therefore it is sensible to research the problem of imprecision assessment.

For any fixed finite space $\mathbb{X}=\left\{x_{1}, x_{2}, \ldots, x_{f}\right\}$, the proper tool for measuring the ambiguity of a fuzzy subset $\mathrm{A} \in \mathcal{F}(\mathbb{X})$ is an energy measure $d: \mathcal{F}(\mathbb{X}) \rightarrow \mathbb{R}_{0}^{+}$, proposed by de Luca and Termini [53], as follows:

$$
d(A)=m(A)=\sum_{i=1}^{f} \mu_{A}\left(x_{i}\right) .
$$

The right tool for measuring the indistinctness is the entropy measure, also proposed by de Luca and Termini [54]. In this article, the entropy measure $e: \mathcal{F}(\mathbb{X}) \rightarrow \mathbb{R}_{0}^{+}$is described as in [55]. For an arbitrary $A \in \mathcal{F}(\mathbb{X})$, we have 


$$
e(A)=\frac{m\left(A \cap A^{C}\right)}{m\left(\left(A \cup A^{C}\right) \cap \mathbb{S}(A)\right)},
$$

where the symbol $\mathbb{S}(A)$ denotes the support of the fuzzy subset.

\section{Oriented Fuzzy Present Value}

The present value (PV) is called the current equivalent value of payments at a fixed point in time. The PV of the future cash flow may be imprecise. For this reason, the PV is described using fuzzy numbers. Then, PV is characterized by a monotonic sequence $\left\{V_{b}, V_{f}, \check{C}, V_{l}, V_{e}\right\}$, where

- $\quad \check{C}$ is the market price.

- $\left[V_{b}, V_{e}\right] \subset \mathbb{R}^{+}$is an interval of all possible PV values.

- $\left[V_{f}, V_{l}\right] \subset\left[V_{s}, V_{e}\right]$ is an interval of all prices that do not perceptibly differ from the market price $\check{C}$.

PV is estimated in an imprecise manner and it supplemented by a forecast of its closest changes. Such a present value is called the oriented present value (OPV). OPV is estimated by OFN:

$$
\overleftrightarrow{P V}=\overleftrightarrow{T r}\left(V_{b}, V_{f}, V_{l}, V_{e}\right)
$$

If $V_{b}<V_{e}$, then the positively oriented PV means the forecast of the value has increased. If $V_{b}<V_{e}$, then the negative orientation means the forecast of the value has decreased.

For example, in [56], shares' OPVs are determined as TrOFNs describing their Japanese candles [57].

Example 1. We evaluate the portfolio $\pi$ composed [56] of:

- $\quad$ a block of shares in Assecopol (ACP);

- a block of shares in ENERGA (ENG);

- a block of shares in JSW (JSW);

- a block of shares in KGHM (KGH);

- a block of shares in LOTOS (LTS);

- a block of shares in ORANGEPL (OPL); and

- $\quad$ a block of shares in PKOBP (PKO).

Based on the closing of the session on the Warsaw Stock Exchange on 15 January 2018, for each considered share, we determine its OPV as a TrOFN describing its Japanese candle. Obtained in this way, shares' OPVs are presented in Table 1.

Table 1. Evaluation of portfolio $\pi$ components.

\begin{tabular}{ccccc}
\hline $\begin{array}{c}\text { Stock } \\
\text { Company }\end{array}$ & Present Value $\overleftrightarrow{P V}_{s}$ & Market Price $\check{C}_{s}$ & $\begin{array}{c}\text { Expected Return } \\
\text { Rate } \overline{\boldsymbol{r}}_{\boldsymbol{s}}\end{array}$ & Variance $\sigma_{s}^{2}$ \\
\hline ACP & $\overleftrightarrow{\mathcal{T} \nabla}(45.90 ; 45.90 ; 45.50 ; 45.48)$ & 45.70 & 0.0300 & 0.000090 \\
CPS & $\overleftrightarrow{\mathcal{T} \nabla}(22.92 ; 22.82 ; 22.82 ; 22.76)$ & 22.82 & 0.0355 & 0.000190 \\
ENG & $\overleftrightarrow{\mathcal{T} \nabla}(10.22 ; 10.19 ; 10.17 ; 10.14)$ & 10.18 & 0.0150 & 0.000020 \\
JSW & $\overleftrightarrow{\mathcal{T} \nabla}(92.24 ; 92.54 ; 92.54 ; 92.80)$ & 92.54 & 0.0400 & 0.000290 \\
KGH & $\overleftrightarrow{\mathcal{T} \nabla}(102.65 ; 103.05 ; 103.60 ; 103.90)$ & 103.33 & 0.0390 & 0.000210 \\
LTS & $\overleftrightarrow{\mathcal{T} \nabla}(56.70 ; 56.56 ; 56.40 ; 56.28)$ & 56.48 & 0.0450 & 0.000390 \\
OPL & $\overleftrightarrow{\mathcal{T} \nabla}(5.75 ; 5.76 ; 5.90 ; 5.90)$ & 5.83 & 0.0360 & 0.000280 \\
PGE & $\overleftrightarrow{\mathcal{T} \nabla}(10.39 ; 10.39 ; 10.35 ; 10.33)$ & 10.37 & 0.0235 & 0.000160 \\
PKO & $\overleftrightarrow{\mathcal{T} \nabla}(42.61 ; 42.61 ; 43.22 ; 43.22)$ & 42.91 & 0.0420 & 0.000370 \\
\hline
\end{tabular}

We notice that:

Source [56] and own elaboration. 
- The companies KGH, JWS, OPL and PKO are evaluated by positively oriented PVs, predicting a rise in market price.

- $\quad$ The companies ACP, CPS, ENG, LTS and PGE are evaluated by negatively oriented PV, predicting a fall in market price.

\section{Oriented Fuzzy Discount Factor}

By the term "financial security", we understand the authorization to receive future financial revenue, payable at some maturity. The value of this revenue is interpreted as an anticipated future value (FV) of the asset. Let us assume that the time horizon $t>0$ of an investment is fixed. According to uncertainty theory $[58,59]$, the future state of affairs is uncertain. Uncertainty theory, as viewed by Mises and Kaplan, is a result of our lack of knowledge about the future state of affairs. However, in the researched case, we can point out a particular time in the future when the considered state of affairs will already be known to the observer. This kind of Mises-Kaplan uncertainty is further referred to as "uncertainty". Considering the work in $[8,9,60-64]$, we accept that this is a sufficient condition for modeling the uncertainty with probability. Thus, uncertainty is often also called quantitative uncertainty. It is worth noting that FV is not burdened by Knight uncertainty [65]. All this leads to the conclusion that FV is a random variable $\widetilde{V}_{t}: \Omega \rightarrow \mathbb{R}$ where the symbol $\Omega$ denotes the space of all states of the financial market.

The security considered here is determined by two values: anticipated FV $=V_{t}$ and assessed $\mathrm{PV}=V_{0}$. The basic characteristic of benefits from owning this security is the simple return rate defined as

$$
r_{t}=\frac{V_{t}-V_{0}}{V_{0}}=\frac{V_{t}}{V_{0}}-1 .
$$

The return rate is a function of FV, which is uncertain by its nature. Currently, we have an extensive knowledge on this subject.

In practice, for financial market analysis, uncertainty is usually described by a probability distribution of the return rate determined by

$$
\widetilde{r}_{t}(\omega)=\frac{\widetilde{V}_{t}(\omega)-\check{C}}{\check{C}}
$$

where the symbol $\check{C}$ denotes the current market price. Following Markowitz [66], we assume that this simple return rate has the Gaussian distribution $N(\bar{r}, \sigma)$.

Example 2. All considerations in the paper are run for the quarterly period of investment time $t=1$ quarter. Parameters of quarterly distribution of return rates of portfolio components $\pi$ are presented in Table 1 .

Moreover, we have

$$
\widetilde{V}_{t}(\omega)=\left(1+\widetilde{r}_{t}(\omega)\right) \check{C} .
$$

Let us consider now the case when PV is determined as OPV $\overleftrightarrow{T r}\left(V_{b}, V_{f}, V_{l}, V_{e}\right)$ represented by

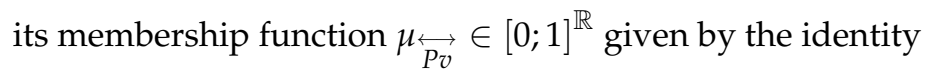

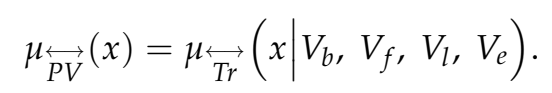

According to the Zadeh's extension principle, the simple return rate calculated for the OFPV is a fuzzy probabilistic set represented by its membership function $\widetilde{\rho} \in[0 ; 1]^{\mathbb{R} \times \Omega}$ given by:

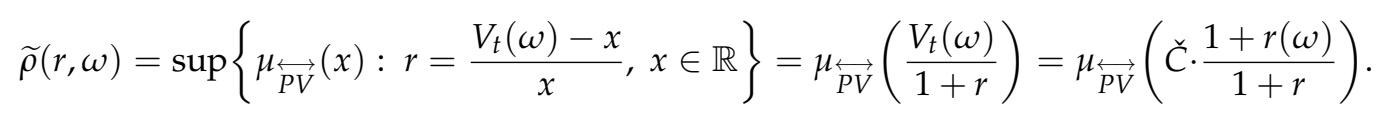


Then, the membership function $\rho \in[0 ; 1]^{\mathbb{R}}$ of the expected return rate is calculated in the following way

$$
\rho(r)=\int_{-\infty}^{+\infty} \mu_{P V}\left(\check{C} \cdot \frac{1+y}{1+r}\right) d F_{r}(y)=\mu_{P V}\left(\check{C} \cdot \frac{1+\bar{r}}{1+r}\right) .
$$

In [47], it is shown that, for appraising the considered securities, the expected fuzzy discount factor is a better tool than the expected fuzzy return rate. Therefore, we determine expected discount factor for the case of OPV. In general, for given return rate $r_{t}$, the discount factor $v_{t}$ is explicitly determined by the identity

$$
v_{t}=\frac{1}{1+r_{t}} \text {. }
$$

We consider expected discount factor (EDF) $\bar{v} \in \mathbb{R}$ defined by identity:

$$
\bar{v}=\frac{1}{1+\bar{r}} .
$$

In agreement with Equation (22), the membership function $\delta \in[0,1]^{\mathbb{R}}$ of an oriented fuzzy EDF (OEDF) $\stackrel{\leftrightarrow}{\mathcal{V}} \in \mathbb{K}$ is given by the identity:

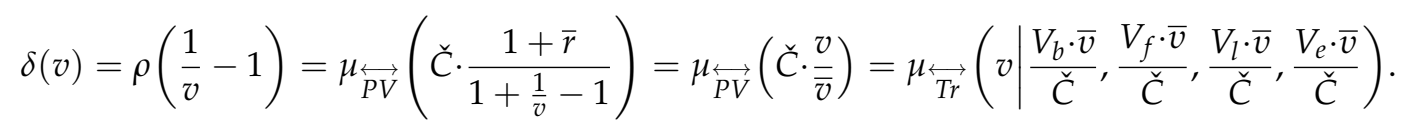

Then, OEDF is given as follows:

$$
\overleftrightarrow{\mathcal{V}}=\overleftrightarrow{\operatorname{Tr}}\left(\frac{V_{b}}{\check{C}} \cdot \bar{v}, \frac{V_{f}}{\check{C}} \cdot \bar{v}, \frac{V_{l}}{\check{C}} \cdot \bar{v}, \frac{V_{e}}{\check{C}} \cdot \bar{v}\right)
$$

\begin{tabular}{|c|c|c|c|}
\hline Stock Company & $\operatorname{EDF} \bar{v}_{s}$ & OEDF $\stackrel{\leftrightarrow}{V}_{s}$ & SPT $H_{s}$ \\
\hline $\mathrm{ACP}$ & 0.9709 & $\overleftrightarrow{\mathcal{T} \nabla}(0.9751 ; 0.9751 ; 0.9666 ; 0.9662)$ & 0.9697 \\
\hline CPS & 0.9657 & $\overleftrightarrow{\mathcal{T} \nabla}(0.9699 ; 0.9657 ; 0.9657 ; 0.9632)$ & 0.9597 \\
\hline ENG & 0.9852 & $\overleftrightarrow{\mathcal{T} \nabla}(0.9891 ; 0.9862 ; 0.9842 ; 0.9813)$ & 0.9816 \\
\hline JSW & 0.9615 & $\overleftrightarrow{\mathcal{T} \nabla}(0.9584 ; 0.9615 ; 0.9615 ; 0.9642)$ & 0.9524 \\
\hline KGH & 0.9625 & $\overleftrightarrow{\mathcal{T} \nabla}(0.9592 ; 0.9599 ; 0.9650 ; 0.9678)$ & 0.9581 \\
\hline LTS & 0.9569 & $\overleftrightarrow{\mathcal{T} \nabla}(0.9606 ; 0.9583 ; 0.9555 ; 0.9535)$ & 0.9461 \\
\hline OPL & 0.9652 & $\overleftrightarrow{\mathcal{T} \nabla}(0.9520 ; 0.9536 ; 0.9768 ; 0.9768)$ & 0.9531 \\
\hline PGE & 0.9770 & $\overleftrightarrow{\mathcal{T} \nabla}(0.9789 ; 0.9789 ; 0.9751 ; 0.9732)$ & 0.9622 \\
\hline PKO & 0.9597 & $\overleftrightarrow{\mathcal{T} \nabla}(0.9530 ; 0.9530 ; 0.9666 ; 0.9666)$ & 0.9474 \\
\hline
\end{tabular}

Example 3. Using Equation (25), we calculate quarterly EDF and OEDF for each component of the portfolio $\pi$. The evaluations obtained in this way are presented in Table 2.

Table 2. Expected discount factors of portfolio $\pi$ components.

The discount factor of a security described in this way is an oriented fuzzy number with the identical orientation as the oriented present value used for estimation. It is worth stressing that the maximum criterion of the expected return rate can be replaced by the minimum criterion of the expected discount factor. In the case of crisp values for both parameters, these criteria are equivalent. 


\section{Investment Recommendations}

The investment recommendation is the counsel given by advisors to investors. In this paper, we consider the family of advice applied in [49]. Therefore, recommendations are expressed by means of standardized advice [49]:

- Buy—suggesting that evaluated security is significantly undervalued;

- Accumulate-suggesting that evaluated security is undervalued;

- Hold-suggesting that evaluated security is fairly valued;

- Reduce-suggesting that evaluated security is overvalued; and

- Sell—suggesting that evaluated security is significantly overvalued.

The above-mentioned advice constitutes the set $\mathbb{A}=\left\{A^{++}, A^{+}, A^{0}, A^{-}, A^{--}\right\}$-called a rating scale where:

- $A^{++}$denotes the advice buy;

- $A^{+}$denotes the advice accumulate;

- $A^{0}$ denotes the advice hold;

- $A^{-}$denotes the advice reduce; and

- $A^{--}$denotes the advice sell.

Due to such approach we will be able to compare the obtained results with the results of similar research conducted in [49].

\subsection{Adviser's Counsel Dependent on Expected Return Rate}

Consider a fixed security $\breve{S}$, represented by the pair $\left(\bar{r}_{s}, \omega_{S}\right)$, where $\bar{r}_{S}$ is an expected return on $\breve{S}$ and $\omega_{S}$ is a parameter characterizing the security $\breve{S}$. An adviser's counsel depends on the expected return. The criterion for a competent choice of advice can be presented as a comparison of the values profit $g\left(\bar{r}_{s} \mid \omega_{s}\right)$ and the profitability threshold (PT) $\breve{G}$, where $g\left(\cdot \mid \omega_{s}\right): \mathbb{R} \rightarrow \mathbb{R}$ is an increasing function of the expected return rate. By the symbol $\mathbb{S}$, we denote the set of all considered securities. In [49], the advice choice function $\Lambda: \mathbb{S} \times \mathbb{R} \rightarrow 2^{\mathbb{A}}$ is defined as follows:

$$
\begin{aligned}
& A^{++} \in \Lambda(\check{S}, \check{G}) \Leftrightarrow g\left(\bar{r}_{s} \mid \omega_{s}\right)>\check{G} \Leftrightarrow g\left(\bar{r}_{s} \mid \omega_{s}\right) \geq \check{G} \wedge \neg g\left(\bar{r}_{s} \mid \omega_{s}\right) \leq \check{G}, \\
& A^{+} \in \Lambda(\check{S}, \check{G}) \Leftrightarrow g\left(\bar{r}_{s} \mid \omega_{s}\right) \geq \check{G}, \\
& A^{0} \in \Lambda(\check{S}, \check{G}) \Leftrightarrow g\left(\bar{r}_{s} \mid \omega_{s}\right)=\check{G} \Leftrightarrow g\left(\bar{r}_{s} \mid \omega_{s}\right) \geq \check{G} \geq \check{G} \wedge g\left(\bar{r}_{s} \mid \omega_{s}\right) \leq \check{G}, \\
& A^{-} \in \Lambda(\check{S}, \check{G}) \Leftrightarrow g\left(\bar{r}_{s} \mid \omega_{s}\right) \leq \check{G}, \\
& A^{--} \in \Lambda(\check{S}, \check{G}) \Leftrightarrow g\left(\bar{r}_{s} \mid \omega_{s}\right)<\check{G} \Leftrightarrow \neg g\left(\bar{r}_{s} \mid \omega_{s}\right) \geq \check{G} \wedge g\left(\bar{r}_{s} \mid \omega_{s}\right) \leq \check{G} .
\end{aligned}
$$

In this way, the advice subset $\Lambda(\breve{S}, \breve{G}) \subset \mathbb{A}$ is assigned, which is interpreted as the investment recommendation given for the security.

\subsection{Adviser's Counsel Dependent on Expected Discount Factor}

In the above section, the investment recommendation depends on the fuzzy expected return. We can assume that a given security $\breve{S}$ is represented by the ordered pair $\left(\bar{v}_{s}, \omega_{s}\right)$ where

$$
\bar{v}_{s}=\frac{1}{1+\bar{r}_{s}}
$$

is EDF for $\breve{S}$. It is very easy to check that we have

$$
g\left(\bar{r}_{s} \mid \mathfrak{\omega}_{s}\right) \geq \check{G} \Leftrightarrow \bar{v}_{s} \leq \frac{1}{1+g^{-1}\left(\check{G} \mid \mathfrak{\omega}_{s}\right)}=H_{s}
$$




$$
g\left(\bar{r}_{s} \mid \varpi_{s}\right) \leq \check{G} \Leftrightarrow \bar{v}_{s} \geq H_{s} .
$$

The value $H_{s}$ is interpreted as a specific profitability threshold (SPT) determined for the security $\check{S}$ Then, the advice choice function $\Lambda: \mathbb{S} \times \mathbb{R} \rightarrow 2^{\mathbb{A}}$ is equivalently described in the following way:

$$
\begin{gathered}
A^{++} \in \Lambda(\check{S}, \check{G}) \Leftrightarrow \bar{v}_{s} \leq H_{s} \wedge \neg \bar{v}_{s} \geq H_{s}, \\
A^{+} \in \Lambda(\check{S}, \check{G}) \Leftrightarrow \bar{v}_{s} \leq H_{s}, \\
A^{0} \in \Lambda(\check{S}, \check{G}) \Leftrightarrow \bar{v}_{s} \leq H_{S} \wedge \bar{v}_{s} \geq H_{s}, \\
A^{-} \in \Lambda(\check{S}, \check{G}) \Leftrightarrow \bar{v}_{s} \geq H_{s}, \\
A^{--} \in \Lambda(\check{S}, \check{G}) \Leftrightarrow \neg \bar{v}_{s} \leq H_{s} \wedge \bar{v}_{s} \geq H_{s} .
\end{gathered}
$$

\subsection{Adviser's Counsel Dependent on Fuzzy Expected Discount Factor}

Now, we consider the case when EDFs are imprecisely evaluated. Moreover, we can additively predict future changes in EDF value. Then, a given security $\breve{S}$ is represented by the ordered pair $\left(\stackrel{\mathcal{V}}{s}_{s}, \omega_{s}\right)$ where

$$
\overleftrightarrow{\mathcal{V}}_{s}=\overleftrightarrow{\operatorname{Tr}}\left(D_{b}^{(s)}, D_{f}^{(s)}, D_{l}^{(s)}, D_{b}^{(s)}\right)
$$

is OEDF calculated using Equation (26) for $\breve{S}$. For this case, using Equation (29), we calculate specific profitability threshold $H_{s}$. If the PT $\breve{G}$ is given, then each SPT $H_{S}$ may be represented by TrOFN

$$
\overleftrightarrow{\llbracket H_{s} \rrbracket}=\overleftrightarrow{\operatorname{Tr}}\left(H_{s}, H_{s}, H_{s}, H_{s}\right)
$$

Then, the value $\widetilde{\Lambda}(\breve{S}, \breve{G})$ of the recommendation choice function $\widetilde{\Lambda}:[0,1]^{\mathbb{R}} \times \mathbb{R} \rightarrow[0,1]^{\mathbb{R}}$ is the membership function $\lambda(\cdot \mid \breve{S}, \breve{G}): \mathbb{A} \rightarrow[0,1]$ function determined in accordance with Equation (31) in the following way:

$$
\begin{aligned}
& \lambda\left(A^{++} \mid \check{S}, \check{G}\right)=v_{Q}\left(\overleftrightarrow{\llbracket H_{s} \rrbracket}, \overleftrightarrow{\mathcal{V}}_{s}\right) \wedge\left(1-v_{Q}\left(\overleftrightarrow{\mathcal{V}}_{s}, \overleftrightarrow{\llbracket H_{s} \rrbracket}\right)\right) \\
& \lambda\left(A^{+} \mid \check{S}, \check{G}\right)=v_{Q}\left(\overleftrightarrow{\llbracket H_{s} \rrbracket}, \overleftrightarrow{\mathcal{V}}_{s}\right) \\
& \lambda\left(A^{0} \mid \check{S}, \check{G}\right)=v_{Q}\left(\overleftrightarrow{\llbracket H_{s} \rrbracket}, \overleftrightarrow{\mathcal{V}}_{s}\right) \wedge v_{Q}\left(\overleftrightarrow{\mathcal{V}}_{s}, \overleftrightarrow{\llbracket H_{s} \rrbracket}\right) \\
& \lambda\left(A^{-} \mid \check{S}, \check{G}\right)=v_{Q}\left(\overleftrightarrow{\mathcal{V}_{s}}, \overleftrightarrow{\llbracket H_{s} \rrbracket}\right) \\
& \lambda\left(A^{--} \mid \check{S}, \breve{G}\right)=v_{Q}\left(\overleftrightarrow{\mathcal{V}}_{s}, \overleftrightarrow{H_{s} \rrbracket}\right) \wedge\left(1-v_{Q}\left(\overleftrightarrow{\llbracket H_{s} \rrbracket}, \overleftrightarrow{\mathcal{V}}_{s}\right)\right)
\end{aligned}
$$

From the point of view of a multivalued logic, the value $\lambda(A \mid \check{S}, \breve{G})$ is interpreted as a logic value of the sentence

"Recommendation $A \in \mathbb{A}$ is advised to take an investment decision".

From the viewpoint of decision-making, the value $\lambda(A \mid \check{S}, \breve{G})$ is interpreted as a degree of recommendation support $A \in \mathbb{A}$, i.e., a declared share of the advisors responsible for a final decision according to the advice $A \in \mathbb{A}$. In the described situation, the investment recommendation $\widetilde{\Lambda}(\breve{S}, \breve{G})$ is the fuzzy subset in the rating scale $\mathbb{A}$. 


\subsection{Recommendation Risk}

This uncertainty stems from an investor's lack of knowledge about the future state of affairs. This lack of knowledge implies that no investor is sure of their future profits or losses. An increase of uncertainty can result in higher risk of choosing a wrong financial decision. This type of risk is called an uncertainty risk. The properties of such risk are discussed in a rich body of literature. In this paper, we evaluate the uncertainty risk burdening the recommendation $A \in \mathbb{A}$ by the variance $\sigma^{2}$ of return rate from considered financial instrument.

The increase in the ambiguity of the recommendation $A \in \mathbb{A}$ suggests a higher number of alternative recommendations to choose from. This leads to an increase in the risk of choosing an incorrect assessment from recommended alternative ones. This may result in making a decision that will be ex post associated with a profit lower than maximal, that is with a chance of loss. This kind of risk is called an ambiguity risk. It is not a coincidence that ambiguity and uncertainty risks have the same description of dangers caused by taken risk. In addition, effects of a decision made under the ambiguity risk are identical to those of a decision made under uncertainty risk. Both risks differ only by their causes. This difference suggests that both risks should be assessed by different methods. In this article, the ambiguity risk burdening the recommendation $A \in \mathbb{A}$ is assessed with energy measure $d(A)$ determined by Equation (14).

An increase in the indistinctness of the recommendation $A \in \mathbb{A}$ suggests that the differences between recommended and not recommended decision alternatives are harder to differentiate. This leads to an increase in the indistinctness risk, that is, the risk of choosing a not recommended option. The indistinctness risk of recommendation $A \in \mathbb{A}$ is measured by the entropy measure $e(A)$ given by Equation (15).

Imprecision risk consists of both ambiguity and indistinctness risks, combined.

\section{Sharpe's Ratio}

Sharpe's ratio is one of the criteria of risk management. In this model of financial equilibrium, the compared values are the expected return on a security and the expected return on the market portfolio. Sharpe's profit index estimates the amount of the premium per overall risk unit. Sharpe's PT is equal to the unit premium of the market portfolio risk $[49,50]$. We assume that there exists a risk-free bond instrument represented by the pair $\left(r_{0}, 0\right)$ and the market portfolio represented by the pair $\left(\bar{r}_{M}, \sigma_{M}\right)$.

Example 4. We focus on the Warsaw Stock Exchange. We consider a financial market with risk-free bound instrument determined as quarterly treasure bonds with return rate $r_{0}=0.0075$. The market portfolio is represented by a portfolio determining stock exchange index WIG20. The return rate from WIG20 is the Gaussian distribution $N\left(r_{M}, \sigma_{M}^{2}\right)=N(0.0200,0.000025)$.

If the security $\check{S}$ is represented by the pair $\left(\bar{r}_{S}, \sigma_{S}\right)$, then, according to Sharp, the profit index $g\left(\cdot \mid \sigma_{S}\right): \mathbb{R} \rightarrow \mathbb{R}$ and the PT $\breve{G}$ are defined as follows:

$$
\begin{gathered}
g\left(\bar{r}_{s} \mid \sigma_{s}\right)=\frac{r_{s}-r_{0}}{\sigma_{s}}, \\
\check{G}=\frac{r_{M}-r_{0}}{\sigma_{M}} .
\end{gathered}
$$

For this case, we calculate SPT $H_{S}$ in following manner:

$$
H_{S}=\frac{\sigma_{M}}{\sigma_{S} \cdot\left(r_{M}-r_{0}\right)+\sigma_{M} \cdot\left(r_{0}+1\right)}
$$

Example 5. Using Equation (38), we calculate SPT for each component of the portfolio $\pi$. Evaluations obtained in this way are presented in Table 2. 
These are ambiguous recommendations. It means that even the use of precise premises does not guarantee obtaining precise recommendations.

\section{Case Study}

In this section, we present recommendations based on Sharpe criterion for portfolio $\pi$ components described in Example 1. Imprecise evaluations of PV and market price of those assets are presented on Table 1.

Table 2 lists the values of EDF, OEDF and SPT determined for each components of portfolio $\pi$. These values are the only premises to formulate the investment recommendations.

If we assume that PV equals the listed market price, then using the Sharp criterion is simply comparing the precise values of EDF and SPT. According to Equation (31), each analyzed action is attributed a chosen recommendation "Reduce" or "Sell".

The replacement of an accurate PV evaluation by its assessment approximated in a more accurate way reflects the essence of the PV. If we now estimate PV with the use of TrOFN presented in Table 1, then using the Sharpe criterion is simply comparing an imprecise OEF with the precise SPT. By means of Equation (34), we then estimate the values of recommendation choice function presented in Table 3. Table 3 also presents information on the imprecision and uncertainty risks that burden individual recommendations. That information is used to choose the recommendation.

Table 3. Imprecise recommendations.

\begin{tabular}{ccccccccc}
\hline & \multicolumn{3}{c}{ Recommendation Choice Function } & \multicolumn{3}{c}{ Risk Evaluation } \\
\hline $\begin{array}{c}\text { Stock } \\
\text { Company }\end{array}$ & $\boldsymbol{A}^{--}$ & $\boldsymbol{A}^{-}$ & $\boldsymbol{A}^{0}$ & $\boldsymbol{A}^{+}$ & $\boldsymbol{A}^{++}$ & Variance & $\begin{array}{c}\text { Energy } \\
\text { Measure }\end{array}$ & $\begin{array}{c}\text { Entropy } \\
\text { Measure }\end{array}$ \\
\hline ACP & 0 & 1 & 1 & 1 & 0 & $\mathbf{0 . 0 0 0 0 9 0}$ & 3 & 0 \\
CPS & 1 & 1 & 0 & 0 & 0 & $\mathbf{0 . 0 0 0 1 9}$ & 2 & 0 \\
ENG & 0.8965 & 1 & 0.1035 & 0.1035 & 0 & $\mathbf{0 . 0 0 0 0 2 0}$ & 2.1035 & 0.0662 \\
JSW & 1 & 1 & 0 & 0 & 0 & $\mathbf{0 . 0 0 0 2 9 0}$ & 2 & 0 \\
KGH & 1 & 1 & 0 & 0 & 0 & $\mathbf{0 . 0 0 0 2 1 0}$ & 2 & 0 \\
LTS & 1 & 1 & 0 & 0 & 0 & $\mathbf{0 . 0 0 0 3 9 0}$ & 2 & 0 \\
OPL & 0.3125 & 1 & 0.6875 & 0.6875 & 0 & $\mathbf{0 . 0 0 0 2 8 0}$ & 2.6875 & 0.2308 \\
PGE & 1 & 1 & 0 & 0 & 0 & $\mathbf{0 . 0 0 0 1 6 0}$ & 2 & 0 \\
PKO & 1 & 1 & 0 & 0 & 0 & $\mathbf{0 . 0 0 0 3 7 0}$ & 2 & 0 \\
\hline
\end{tabular}

Investment recommendations for ACP, ENG and OPL are burdened with increased ambiguity risk. Moreover, the recommendations for ENG and OPL carry indistinctness risk. For that reason, those recommendations are rejected. Eventually, only the following stocks are attributed with "Reduce" or "Sell" recommendation: CPC, JSW, KGHM, LTS, PGE and PKO.

Thus, the disclosure of imprecision of PV estimations allows rejecting riskier recommendations.

Another example of recommendation choice function use can be the assistance in investment decisions regarding a single entity. For instance, an investor considering an investment strategy regarding the entity ENG obtains the following pieces of advice:

- The most advisable is the investment decision "Reduce".

- The investment decision "Sell" is not much worse.

- The investment decisions "Hold" or "Accumulate" cannot be excluded.

- The investment decision "Buy" should be rejected.

In turn, the final decision is taken by the investor. Their personal responsibility for taking this investment decision decreases along with the increase of the value of recommendation choice function. 


\section{Conclusions}

In the paper, it is indicated that, if the premise to formulate an investment recommendation uses the expected oriented fuzzy discount factor, then the recommendation itself is a fuzzy subset in a rating scale.

This way, an investment recommendation form identical to the investment recommendation in [49] was achieved. Additionally, the estimates of imprecision risk burdening the recommendations were obtained. The disclosure of imprecision risk can significantly influence the choice of appropriate investment recommendations. That choice is up to the investors. Those investors choose the final investment decisions. The values of the membership function of investment recommendations should be interpreted as a degree of a chosen advice recommendation, i.e., the declared involvement of the advisor in the responsibility of taking the final investment decision.

As proven in the case study, the proposed imprecise recommendations can be an efficient premise to manage a homogenous package of stocks. At the same time, in the analyzed case study, a method is proposed to choose the offered recommendations to manage the financial portfolio.

The obtained results encourage undertaking further research on the possibility of using other criteria to evaluate the investment decisions to formulate the imprecise recommendations described in Section 6.3. A topic of further research should be the determination of such successive investment strategies for which the oriented fuzzy discount factor is a basic premise for making investment decisions.

In the future, this would enable examining the impact of the discount factor orientation on the form of the investment recommendation.

Funding: This research received no external funding.

Acknowledgments: The author is very grateful to the anonymous reviewers for their insightful and constructive comments and suggestions.

Conflicts of Interest: The author declares no conflict of interest.

\section{References}

1. Dubois, D.; Prade, H. Operations on fuzzy numbers. Int. J. Syst. Sci. 1978, 9, 613-629. [CrossRef]

2. Kosiński, W.; Słysz, P. Fuzzy numbers and their quotient space with algebraic operations. Bull. Pol. Acad. Sci. 1993, 41, 285-295.

3. Kosiński, W.; Prokopowicz, P.; Ślęzak, D. Fuzzy Numbers with Algebraic Operations: Algorithmic Approach; Klopotek, M., Wierzchoń, S.T., Michalewicz, M., Eds.; Physica Verlag: Heidelberg, Germany, 2002; pp. 311-320.

4. Kosiński, W.; Prokopowicz, P.; Ślęzak, D. Ordered fuzzy numbers. Bull. Pol. Acad. Sci. 2003, 51, 327-339.

5. Kosiński, W. On fuzzy number calculus. Int. J. Appl. Math. Comput. Sci. 2006, 16, 51-57.

6. Prokopowicz, P.; Prokopowicz, P.; Czerniak, J.; Mikołajewski, D.; Apiecionek, Ł.; Slezak, D. (Eds.) Theory and Applications of Ordered Fuzzy Number. Tribute to Professor Witold Kosinski; Studies in Fuzziness and Soft Computing, 356; Springer Verlag: Berlin, Germany, 2017. [CrossRef]

7. Piasecki, K. Revision of the Kosiński's Theory of Ordered Fuzzy Numbers. Axioms 2018, 7. [CrossRef]

8. Kolmogorov, A.N. Grundbegriffe der Wahrscheinlichkeitsrechnung; Julius Springer: Berlin, Germany, 1933. [CrossRef]

9. Kolmogorov, A.N. Foundations of the Theory of Probability; Chelsea Publishing Company: New York, NY, USA, 1956.

10. Piasecki, K. Basis of financial arithmetic from the viewpoint of the utility theory. Oper. Res. Dec. 2012, 22, 37-53. [CrossRef]

11. Ward, T.L. Discounted fuzzy cash flow analysis. Fall Ind. Eng. Conf. Proc. 1985, 476-481. [CrossRef]

12. Buckley, J.J. The Fuzzy Mathematics of Finance. Fuzzy Sets Syst. 1987, 21, 257-273. [CrossRef]

13. Greenhut, J.G.; Norman, G.; Temponi, C.T. Towards a fuzzy theory of oligopolistic competition. IEEE Proc. Isuma-Nafips 1995, 286-291. [CrossRef] 
14. Sheen, J.N. Fuzzy financial profitability analyses of demand side management alternatives from participant perspective. Inf. Sci. 2005, 169, 329-364. [CrossRef]

15. Gutierez, I. Fuzzy numbers and Net Present Value. Scand. J. Mgmt. 1989, 5, 149-159. [CrossRef]

16. Kuchta, D. Fuzzy capital budgeting. Fuzzy Sets Syst. 2000, 111, 367-385. [CrossRef]

17. Lesage, C. Discounted cash-flows analysis. An interactive fuzzy arithmetic approach. Eur. J. Econ. Soc. Syst. 2001, 15, 49-68. [CrossRef]

18. Huang, X. Two new models for portfolio selection with stochastic returns taking fuzzy information. Eur. J. Oper. Res. 2007, 180, 396-405. [CrossRef]

19. Tsao, C.T. Assessing the probabilistic fuzzy Net Present Value for a capital, Investment choice using fuzzy arithmetic. J. Chin. Ins. Ind. Eng. 2005, 22, 106-118. [CrossRef]

20. Calzi, M.L. Towards a general setting for the fuzzy mathematics of finance. Fuzzy Sets Syst. 1990, 35, $265-280$. [CrossRef]

21. Piasecki, K. Behavioural present value. Ssrn Electron. J. 2011, 1, 1-17. [CrossRef]

22. Baerecke, T.; Bouchon-Meunier, B.; Detyniecki, M. Fuzzy present value. In Proceedings of the 2011 IEEE Symposium Computational Intelligence for Financial Engineering and Economics, Paris, France, 11-15 April 2011; pp. 1-6.

23. Biswas, P.; Pramanik, S. Fuzzy approach to replacement problem with value of money changes with time. Int. J. Comput. Appl. 2011, 30, 28-33. [CrossRef]

24. Nosratpour, M.; Nazeri, A.; Meftahi, H. Fuzzy net present value for engineering analysis. Manag. Sci. Lett. 2012, 2, 2153-2158. [CrossRef]

25. Piasecki, K. Effectiveness of securities with fuzzy probabilistic return. Oper. Res. Decis. 2011, $21,65-78$.

26. Li, X.; Quin, Z.; Kar, S. Mean-Variance-Skewness model for portfolio selection with fuzzy returns. Eur. J. Oper. Res. 2010, 202, 239-247. [CrossRef]

27. Quin, Z.; Wen, M.; Gu, C. Mean-absolute deviation portfolio selection model with fuzzy returns. Iran. J. Fuzzy Syst. 2011, 8, 61-75.

28. Tsaur, R. Fuzzy portfolio model with different investor risk attitudes. Eur. J. Oper. Res. 2013, 227, $385-390$. [CrossRef]

29. Tanaka, H.; Guo, P.; Turksen, B. Portfolio selection based on fuzzy probabilities and possibility distributions. Fuzzy Sets Syst. 2000, 111, 387-397. [CrossRef]

30. Duan, L.; Stahlecker, P. A portfolio selection model using fuzzy returns. Fuzzy Opt. Dec. Mak. 2011, 10, 167-191. [CrossRef]

31. Guo, H.; Sun, B.; Karimi, H.R.; Ge, Y.; Jin, W. Fuzzy investment portfolio selection models based on interval analysis approach. Math. Probl. Eng. 2012. [CrossRef]

32. Gupta, P.; Mittal, G.; Mehlawat, M.K. Multiobjective expected value model for portfolio selection in fuzzy environment. Optim. Lett. 2013, 7, 1765-1791. [CrossRef]

33. Gupta, P.; Mittal, G.; Saxena, A. Asset portfolio optimization using fuzzy mathematical programming. Inf. Sci. 2008, 178, 1734-1755. [CrossRef]

34. Huang, X.; Shen, W. Multi-period mean-variance model with transaction cost for fuzzy portfolio selection. In Proceedings of the Seventh International Conference on Fuzzy Systems and Knowledge Discovery, Yantai, Shandong, China, 10-12 August 2010. [CrossRef]

35. Liu, Y.; Sun, L. Comparative Research of Portfolio Model Using Fuzzy Theory. In Proceedings of the Fourth International Conference on Fuzzy Systems and Knowledge Discovery, Haikou, China, 24-27 August 2007. [CrossRef]

36. Liu, Y.J.; Zhang, W.G. Fuzzy portfolio optimization model under real constraints. Insur. Math. Econ. 2013, 53, 704-711. [CrossRef]

37. Wu, X.L.; Liu, Y.K. Optimizing fuzzy portfolio selection problems by parametric quadratic programming. Fuzzy Optim. Decis. Mak. 2012, 11, 411-449. [CrossRef]

38. Wang, S.; Zhu, S. On fuzzy portfolio selection problems. Fuzzy Optim. Decis. Mak. 2002, 1, 361-377. [CrossRef]

39. Mehlawat, M.K. Credibilistic mean-entropy models for multi-period portfolio selection with multi-choice aspiration levels. Inf. Sci. 2016, 345, 9-26. [CrossRef]

40. Kahraman, C.; Ruan, D.; Tolga, E. Capital budgeting techniques using discounted fuzzy versus probabilistic cash flows. Inf. Sci. 2002, 142, 57-76. [CrossRef] 
41. Fang, Y.; Lai, K.K.; Wang, S. Fuzzy Portfolio Optimization: Theory and Methods; Lecture Notes in Economics and Mathematical Systems; Springer: Berlin, Germany, 2008; Volume 609.

42. Gupta, P.; Mehlawat, M.K.; Inuiguchi, M.; Chandra, S. Fuzzy Portfolio Optimization: Advances in Hybrid Multi-criteria Methodologies; Studies in Fuzziness and Soft Computing; Springer: Berlin, Germany, 2014; Volume 316. [CrossRef]

43. Kosko, B. Fuzziness vs probability. Int. J. Gen. Syst. 1990, 17, 211-240. [CrossRef]

44. Hiroto, K. Concepts of probabilistic sets. Fuzzy Sets Syst. 1981, 5, 31-46. [CrossRef]

45. Hao, F.F.; Liu, Y.K. Portfolio Selection Problem in Fuzzy Random Decision Systems. In Proceedings of the 3rd International Conference on Innovative Computing Information and Control, Dalian, Liaoning, China, 11-14 July 2008. [CrossRef]

46. Hasuike, T.; Katagiri, H.; Ishii, H. Portfolio selection problems with random fuzzy variable returns. In Proceedings of the 2007 IEEE International Fuzzy Systems Conference, London, UK, 23-26 July 2007. [CrossRef]

47. Piasecki, K.; Siwek, J. Two-Asset Portfolio with Triangular Fuzzy Present Values-An Alternative Approach; Contemporary Trends in Accounting, Finance and Financial Institutions. Proceedings from the International Conference on Accounting, Finance and Financial Institutions (ICAFFI), Poznan 2016; Choudhry, T., Mizerka, J., Eds.; Springer International Publishing: Berlin, Germany, 2018; pp. 11-26. [CrossRef]

48. Siwek, J. Multiple asset portfolio with present value given as a discrete fuzzy number. MME 2017, 2017, 661-666.

49. Piasecki, K. On Imprecise Investment Recommendations. Stud. Logic Gramm. Rhetor. 2014, 37, $179-194$. [CrossRef]

50. Sharpe, W.F. Mutual fund performance. J. Bus. 1966, 19, 119-138. [CrossRef]

51. Dubois, D.; Prade, H. Fuzzy real algebra: Some results. Fuzzy Set. Syst. 1979, 2, 327-348. [CrossRef]

52. Klir, G.J. Developments in uncertainty-based information. Adv. Comput. 1993, 36, 255-332.

53. De Luca, A.; Termini, S. A definition of a non-probabilistic entropy in the settings of fuzzy set theory. Inf. Control 1972, 20, 301-312. [CrossRef]

54. De Luca, A.; Termini, S. Entropy and energy measures of fuzzy sets. In Advances in Fuzzy Set Theory and Applications; Gupta, M.M., Ragade, R.K., Yager, R.R., Eds.; North-Holland: Amsterdam, The Netherlands, 1979; pp. 321-338.

55. Kosko, B. Fuzzy entropy and conditioning. Inf. Sci. 1986, 40, 165-174. [CrossRef]

56. Łyczkowska-Hanćkowiak, A.; Piasecki, K. On representation of Japanese candlesticks by ordered fuzzy numbers. In 9th International Scientific Conference "Analysis of International Relations 2018. Methods and Models of Regional Development. Winter Edition"; Szkutnik, W., Ed.; University of Economics in Katowice Publishing: Katowice, Poland, 2018; pp. 61-68.

57. Nison, S. Japanese Candlestick Charting Techniques; New York Institute of Finance: New York, NY, USA, 1991.

58. Von Mises, L. The Ultimate Foundation of Economic Science; An Essay on Method; Liberty Fund: Carmel, IN, USA, 1962.

59. Kaplan, S.; Barish, N.N. Decision-Making Allowing Uncertainty of Future Investment Opportunities. Manag. Sci. 1967, 13, 569-577. [CrossRef]

60. Von Mises, L. Probability, Statistics and Truth; Dover Publications: Mineola, NY, USA, 1957. Available online: https:/ / www.maa.org/press/maa-reviews/probability-statistics-and-truth (accessed on 24 February 2019).

61. Von Lambalgen, M. Randomness and foundations of probability: Von Mises' axiomatization of random sequences. Inst. Math. Stat. Lect. Notes Monogr. Ser. 1996, 30, 347-367.

62. Sadowski, W. Forecasting and Decision Making. Quantitative Wirtschafts-und Unternehmensforschung; Springer: Berlin/Heidelberg, Germany, 1980.

63. Czerwiński, Z. Enumerative induction and the theory of games. Studia Log. 1960, 10, 29-38. [CrossRef]

64. Caplan, B. Probability, common sense, and realism: A reply to Hulsmann and Block. Q. J. Austrian Econ. 2001, 4, 69-86. 
65. Knight, F.H. Risk, Uncertainty, and Profit. Hart, Schaffner E Marx; Houghton Mifflin Company: Boston, MA, USA, 1921.

66. Markowitz, H. Portfolio selection. J. Financ. 1952, 7, 77-91. [CrossRef]

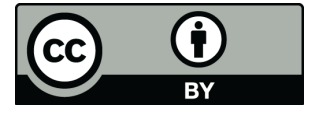

(c) 2019 by the author. Licensee MDPI, Basel, Switzerland. This article is an open access article distributed under the terms and conditions of the Creative Commons Attribution (CC BY) license (http:/ / creativecommons.org/licenses/by/4.0/). 